\title{
Facoemulsificação versus extração extracapsular manual do cristalino: análise de custos
}

\author{
Phacoemulsification and manual extracapsular extraction:costs analysis
}

\author{
Newton Kara-José Junior ${ }^{1}$ \\ Amaryllis Avakian² \\ Leda Mine Takei Lower ${ }^{2}$ \\ Ana Maria Rocha ${ }^{3}$ \\ Marcio Cursino ${ }^{3}$ \\ Milton Ruiz Alves ${ }^{4}$
}

Universidade de São Paulo - Faculdade de Medicina Hospital das Clínicas - Departamento de Oftalmologia Av. Dr. Éneas de Carvalho Aguiar 255 - 6ำ andar São Paulo (SP) CEP 05403-010

${ }^{1}$ Doutor em Oftalmologia e Coordenador do Setor de Catarata do Departamento de Oftalmologia da Universidade de São Paulo - USP.

${ }^{2}$ Doutora em Oftalmologia e Coordenadora do Setor de Catarata do Departamento de Oftalmologia da Universidade de São Paulo - USP.

${ }^{3}$ Médica(o) Estagiária(o) do Setor de Catarata do Departamento de Oftalmologia da Universidade de São Paulo USP.

${ }^{4}$ Professor Livre Docente pela Faculdade de Medicina e Chefe do Setor de Córnea do Departamento de Oftalmologia da Universidade de São Paulo - USP.

Endereço para correspondência: Newton Kara-José Jr. Rua Santa Justina 215 ap 91. São Paulo (SP), CEP 04545-041

E-mail: msffonseca@ hotmail.com

Recebido para publicação em 25.04.2003

Versão revisada recebida em 19.09.2003 Aprovação em 22.03.2004

Cada autor declara que ele/ela não possui interesse financeiro no desenvolvimento ou marketing dos materiais referidos no estudo.

\section{RESUMO}

Objetivo: Avaliar aspectos clínicos e econômicos da cirurgia de catarata pela técnica de facoemulsificação, quando comparada à técnica de extração extracapsular do cristalino no Hospital das Clínicas da Faculdade de Medicina da Universidade de São Paulo. Métodos: Estudo prospectivo em que foram selecionados consecutivamente 162 pacientes, portadores de catarata senil, atendidos em projetos comunitários para identificação e posterior tratamento da catarata (Projetos-Catarata), que atenderam aos critérios de inclusão para o estudo. Esses projetos foram realizados pelo Hospital das Clínicas, entre outubro de 2001 e junho de 2002. Os pacientes selecionados foram randomizados para comporem dois grupos, que seriam submetidos à cirurgia de catarata pela técnica de facoemulsificação e de extração extracapsular. Resultados: Nas condições do estudo, a técnica de facoemulsificação permitiu o uso de anestesia tópica, reduziu o número de retornos e o tempo de duração do período pós-operatório. A utilização da facoemulsificação tornou a cirurgia mais rápida, otimizou o centro cirúrgico e apresentou vantagens econômicas para o hospital. Conclusão: No tocante às condições do Hospital das Clínicas, os custos totais da cirurgia de catarata mediante a técnica de facoemulsificação são maiores do que os custos com a técnica de extração extracapsular; e a remuneração efetuada pelo Sistema Único de Saúde para a cirurgia por facoemulsificação confere maiores vantagens econômicas ao hospital do que a cirurgia por extração extracapsular.

Descritores: Extração de catarata/métodos; Extração de catarata/economia; Facoemulsificação/métodos; Facoemulsificação/economia; Cegueira/prevenção e controle; Fatores econômicos, Hospitais de ensino; Estudo comparativo

\section{INTRODUÇÃO}

A catarata é considerada a principal causa de cegueira, sendo responsável por cerca da metade do número de cegos no mundo. A cegueira por catarata incapacita o indivíduo, aumenta sua dependência, reduz sua condição social e resulta em aposentadoria profissional precoce ${ }^{(1-5)}$. A recuperação da visão pela cirurgia de catarata produz benefícios econômicos e sociais para o indivíduo, sua família e comunidade ${ }^{(6-13)}$.

Foram realizadas, no Brasil, entre 1996 e 1998 e financiadas pelo Sistema Único de Saúde (SUS), em média, 132 mil cirurgias de catarata por ano, proporção essa, insuficiente até mesmo para compensar os novos casos surgidos nesse período ${ }^{(6,14-17)}$.

Alguns autores mostram que para a solução da problemática da cegueira por catarata no Brasil, não é suficiente apenas oferecer o tratamento, mas é necessário, também, adotar medidas educativas junto à população. Faz-se 
necessário a criação de condições que facilitem o acesso à cirurgia, pois, mesmo que esta seja gratuita, o paciente não estará isento de gastos financeiros nos dias das consultas ${ }^{(18-19)}$.

Em 1998 foi instituída a Campanha Nacional de Catarata, mobilizando mais de 3.500 oftalmologistas, que realizaram na rede pública de saúde, nos 3 anos subseqüentes, uma média anual de 263 mil cirurgias de catarata, praticamente o dobro do que era realizado nos anos anteriores. Para que esse número adicional de cirurgias fosse possível, bastou o Projeto-Catarata facilitar, em todo o país, o acesso ao tratamento. Ao Governo Federal, coube permitir que o financiamento das cirurgias pelo SUS fosse em forma de "extra-teto" para as instituições credenciadas $^{(15,20)}$. Na organização desse projeto, foi importante a experiência adquirida previamente pela UNICAMP, que havia estudado e introduzido em hospital público o uso rotineiro de cirurgia ambulatorial sob anestesia local, com otimização do centro cirúrgico ambulatorial ${ }^{(21-29)}$.

O Serviço de Oftalmologia do HCFMUSP, por exemplo, elevou seu número de médicos residentes de 5 para 14, ao ano, e aumentou o número de cirurgias de catarata realizadas anualmente de 836 em 1998 para 3.868 em 2002, graças a características de gerenciamento do Serviço de Oftalmologia. Estimulou a produtividade dos médicos e do pessoal auxiliar envolvido no atendimento, otimizou o centro cirúrgico e facilitou o acesso da população necessitada ao hospital, por meio de mutirões continuados, para triagem (Projeto-Catarata) (Universidade de São Paulo, 2002).

Atualmente a cirurgia de catarata com implante de LIO é um dos procedimentos cirúrgicos mais realizados no mundo, graças aos benefícios conferidos aos operados ${ }^{(30-33)}$. As técnicas mais utilizadas, no momento, para a cirurgia de catarata são a extração extracapsular manual do cristalino (EECP) e a facoemulsificação (FACO).

No momento atual, a FACO é a técnica mais utilizada na maioria dos países desenvolvidos, devido à possibilidade de rápida recuperação visual e ao reduzido índice de complicações per e pós-operatórias ${ }^{(34-35)}$.

Nos Estados Unidos, em 1998, aproximadamente 94\% dos cirurgiões usavam a técnica de FACO em, pelo menos, 95\% das cirurgias de catarata realizadas. No Reino Unido, em 1998, $77 \%$ das cirurgias de catarata realizadas no sistema de saúde nacional foram por $\mathrm{FACO}^{(34-35)}$. Na Suécia, em 2000, 98\% das cirurgias de catarata foram por $\mathrm{FACO}^{(36)}$.

No Brasil e em outros países sul-americanos, ainda não se dispõe desses indicadores atualizados. Acredita-se que os gastos para aquisição e manutenção dos aparelhos e insumos necessários, talvez seja o fator mais limitante para que a técnica de FACO seja difundida nos países em desenvolvimento ${ }^{(15)}$.

Em um hospital universitário da Espanha, os gastos totais (despesas com ato cirúrgico e retornos pós-operatórios) com a utilização da técnica de FACO foram 4,87\% maiores do que os gastos com a técnica de $\operatorname{EECP}^{(37-39)}$. Em estudo realizado na Inglaterra, demonstrou que os maiores gastos com o ato cirúrgico, quando da utilização da técnica de FACO eram compensados pelos reduzidos gastos durante o período pós-operatório ${ }^{(32)}$.
Apesar da cirurgia de catarata ser o procedimento mais realizado nos indivíduos com mais de 65 anos de idade, sendo seus custos responsáveis por parcela significativa dos gastos públicos com saúde, a única pesquisa disponível sobre a exequiibilidade dessa cirurgia em hospitais públicos de países em desenvolvimento é de 1994 e considera a técnica de $\operatorname{EECP}^{(20)}$.

Este estudo prospectivo e randomizado, realizado no HCFMUSP, avalia, em um hospital de ensino, os aspectos econômicos da cirurgia de catarata por FACO e por EECP.

\section{MÉTODOS}

Foi realizado estudo clínico randomizado no Hospital das Clínicas da Faculdade de Medicina da Universidade de São Paulo (HCFMUSP).

Foram estudados aspectos clínicos e econômicos associados a duas técnicas cirúrgicas de extração de catarata senil e seus períodos de recuperação pós-cirúrgico, em pacientes atendidos por campanhas comunitárias, denominadas Projeto-Catarata, realizadas pelo HCFMUSP.

\section{Variáveis}

\section{Aspectos clínico-cirúrgicos (das diferentes técnicas de extração da catarata)}

- tipos de anestesia;

- necessidade de suturas;

- tempo de permanência do paciente na sala cirúrgica;

- tempo de duração da etapa da consulta pós-operatória realizada exclusivamente pelo oftalmologista;

- necessidade de remoção de sutura;

- necessidade de se realizar consulta em data não programada;

\section{Aspectos econômicos (dos procedimentos cirúrgicos)}

- gastos com a manutenção do centro cirúrgico (custos fixos);

- custo da depreciação dos equipamentos necessários para ambas as técnicas cirúrgicas;

- gastos com os insumos utilizados para as cirurgias, em ambas as técnicas (custos variáveis); e gastos com o acompanhamento pós-operatório (em ambas as técnicas).

\section{População e amostra}

Foram selecionados consecutivamente 162 pacientes, portadores de catarata senil, atendidos em projetos comunitários para identificação e posterior tratamento da catarata (Projetos-Catarata), que atenderam aos critérios de inclusão para o estudo. Esses projetos foram realizados pelo HCFMUSP, entre outubro de 2001 e junho de 2002. Dentre os casos selecionados, foram submetidos à cirurgia de catarata, no HCFMUSP, 127 pacientes, que estavam clinicamente aptos para o procedimento e que compareceram ao hospital na data agendada para a cirurgia. 


\section{Critérios de inclusão e de exclusão}

Para compor a amostra, obedeceu-se aos critérios apresentados a seguir:

a) Critérios de inclusão:

- pessoas de ambos os sexos, entre 41 e 80 anos, portadores de catarata senil, com baixa acuidade visual bilateral (AV inferior a 20/40 - escala optométrica de Snellen, no olho de melhor visão, com a melhor correção óptica);

- distância de moradia menor do que $100 \mathrm{~km}$ do HCFMUSP;

- pressão intra-ocular (PIO) inferior a $21 \mathrm{mmHg}$ (sem tratamento) na avaliação inicial; e

- capacidade de compreender e responder ao questionário a ser aplicado.

b) Critérios de exclusão:

- restrições físicas ou clínicas, além do problema visual, capazes de limitar o desempenho do indivíduo para as atividades da vida diária (AVD) e profissional (AVP);

- alterações oculares, além da catarata, que contribuíssem para a diminuição da acuidade visual;

- cirurgias oculares prévias;

- história clínica sugestiva de ambliopia; e

- recusa em participar do estudo e assinar o Termo de Consentimento.

\section{Formação dos grupos}

Os pacientes foram divididos, por sorteio, em dois grupos:

Grupo 1 (composto por 68 indivíduos): pacientes submetidos à cirurgia de catarata por meio da técnica de facoemulsificação (FACO), acrescida do implante de lente intra-ocular (LIO) de câmara posterior; e

Grupo 2 (composto por 59 indivíduos): pacientes submetidos à cirurgia de catarata por meio da técnica de extração extracapsular manual do cristalino (EECP), acrescida do implante de lente intra-ocular (LIO) de câmara posterior.

\section{Randomização}

No momento de sua inclusão no estudo, cada paciente recebeu um número, de acordo com a seqüência de seleção. A seguir, utilizando-se sorteio aleatório, foi realizada a alocação de cada número a um determinado grupo, a fim de evitar qualquer direcionamento de paciente para algum grupo específico. Os médicos envolvidos no estudo não participaram do sorteio dos grupos, mas tiveram conhecimento da modalidade cirúrgica a ser realizada momentos antes da cirurgia.

Os pacientes selecionados que não compareceram à cirurgia foram excluídos do estudo. Os pacientes que tiveram o procedimento suspenso por falta de condições clínicas foram encaminhados para tratamento específico e, conforme resultado da avaliação clínica, ou foram re-agendados para a cirurgia ou excluídos do estudo.

\section{Procedimentos e técnicas}

Os pacientes foram submetidos à anestesia trinta minutos antes do início da operação. Os pacientes a serem submetidos à FACO foram avaliados pela equipe cirúrgica, quanto à possibilidade da cirurgia ser realizada com anestesia tópica. Após explicação sobre as características da anestesia tópica e do bloqueio peribulbar, o paciente optou pelo tipo de anestesia a ser realizada. Os pacientes que demonstrassem intranqüilidade e incapacidade de colaboração durante a cirurgia foram submetidos ao bloqueio peribulbar. Todas as EECPs foram realizadas com bloqueio peribulbar.

Para nenhum caso foi indicada sedação anestésica ou utilizada medicação ansiolítica, segundo a rotina do Centro Cirúrgico Ambulatorial do HCFMUSP.

Para pacientes submetidos à FACO, os retornos após a cirurgia foram realizados no período pós-operatório (PO) de 1 a 2 dias, 7 a 13 dias e 21 a 27 dias (data da provável alta do acompanhamento). A correção visual definitiva, quando necessária, foi prescrita no momento da alta, época em que se considera o erro refrativo estabilizado ${ }^{(40-43)}$.

Para os pacientes submetidos à EECP, os retornos após a cirurgia ocorreram nos PO de 1 a 2 dias, PO de 7 a 13 dias, PO de 28 a 34 dias, PO de 42 a 48 dias e PO de 56 a 62 dias (data da provável alta do acompanhamento). Caso fosse necessário, as suturas eram removidas a partir do PO de 42 a 48 dias, sendo, nesses casos, um retorno adicional agendado para a semana seguinte, a fim de se avaliar o efeito da remoção da sutura sobre o astigmatismo corneal induzido e, se fosse preciso, fazer a remoção de outra sutura. A correção visual, quando necessária, era prescrita no momento da alta, época em que o erro refratométrico fora estabilizado.

\section{Indicadores econômicos relacionados à cirurgia e ao acompanhamento pós-operatório}

Foram considerados, para a estimativa dos custos da cirurgia de catarata, em ambos os grupos (FACO e EECP), no HCFMUSP, em 2002, os seguintes indicadores:

- insumos utilizados para a cirurgia em ambos os grupos (FACO e EECP) - custos variáveis;

- custo operacional do centro cirúrgico e do ambulatório em ambos os grupos (FACO e EECP) - custos fixos; e

- custo de depreciação da aparelhagem cirúrgica necessária em ambos os grupos (FACO e EECP).

\section{Estimativa dos custos variáveis da cirurgia}

Para a estimativa dos custos variáveis das cirurgias, foram inferidas as despesas com os insumos utilizados para cada procedimento, conforme descrição a seguir:

\section{Grupo EECP}

Insumos utilizados para o preparo do paciente e da equipe cirúrgica.

Insumos utilizados para a limpeza da sala cirúrgica.

Insumos utilizados para a anestesia.

Insumos utilizados para a cirurgia, dentre estes:

- substância viscoelástica de hidroxipropilmetilcelulose a $2 \%$

- LIO de PMMA peça única, rígida (13,0 mm de tamanho e 6,0 mm de zona óptica) 


\section{Grupo FACO}

Insumos utilizados para o preparo do paciente e da equipe cirúrgica.

Insumos utilizados para a limpeza da sala cirúrgica.

Insumos utilizados para a cirurgia, dentre estes:

- substância viscoelástica de hidroxipropilmetilcelulose a $2 \%$

- LIO de acrílico hidrofóbico dobrável de 3 peças (12,5 mm de tamanho e 5,5 mm de zona óptica)

- cassete de facoemulsificador (Kit Max Vac), compatível

- para o aparelho Legacy: Alcon (1 cassete para cada ci-

- rurgia)

\section{Estimativa dos custos fixos do Centro Cirúrgico Ambulatorial - Hospital das Clínicas da Faculdade de Medicina da Universidade de São Paulo (HCFMUSP), 2002}

O Centro Cirúrgico Ambulatorial (CCA) - HCFMUSP possui área útil de $250,71 \mathrm{~m}^{2}$, com 5 salas cirúrgicas e funcionamento de 5 dias por semana, 12 horas por dia, sendo que a primeira e a última hora de funcionamento são reservadas, respectivamente, para admissão e preparo do paciente e para limpeza do local. Portanto, o período disponível para a realização das cirurgias é de 10 horas.

Para a estimativa dos custos fixos mensais do CCA HCFMUSP, foram inferidas despesas com:

a) recursos humanos: salários, encargos e benefícios da equipe não médica do CCA-HCFMUSP, composta por 1 enfermeira, 8 auxiliares de enfermagem, 1 auxiliar de escritório e 4 auxiliares de limpeza.

b) espaço físico e consumo de água e energia elétrica: O CCA HCFMUSP não possui gastos diretos com a utilização do espaço físico e com o consumo de água e energia elétrica. Estas despesas são custeadas e diluídas pelo custo operacional do "Complexo Hospitalar". Para a estimativa destes valores, foi considerado o custo proporcional de locação e de consumo de água e energia elétrica de um imóvel de $128 \mathrm{~m}^{2}$, em que funciona uma unidade cirúrgica, situada na mesma região que o HCFMUSP.

c) depreciação de equipamentos: de acordo com a Taxa de Depreciação de Bens, elaborada por FENACON; IOB (2000), instrumentos e aparelhos para cirurgia apresentam prazo de vida útil de 10 anos (taxa de depreciação anual de $10 \%$ ). Considerou-se o valor de mercado, no momento, dividido pelo número de meses (120 meses) de vida útil de um microscópio cirúrgico (Zeiss S5), utilizado para as cirurgias de ambos os grupos e de um facoemulsificador (Legacy - Alcon Laboratórios), utilizado para as cirurgias do grupo FACO.

O custo diário de uma sala do CCA-HCFMUSP foi inferido, dividindo-se os custos fixos mensais estimados pelo número de salas existentes no local (5 salas) e de dias de funcionamento no mês (20 dias). A este valor, foi adicionado despesas com salários, encargos e benefícios de 4 médicos oftalmologistas (2 médicos assistentes do HCFMUSP e 2 médicos residentes do HCFMUSP), revezando-se em duplas, por períodos de 6 horas por dia, utilizando uma sala cirúrgica. Dividindo-se o valor encontrado pelo número de cirurgias realizadas por dia, em uma sala cirúrgica, em ambos os grupos (FACO e EECP), foi estimado o custo fixo de cada procedimento, em ambos os grupos (FACO e EECP).

\section{Estimativa dos custos fixos do Ambulatório de Oftalmologia - HCFMUSP, 2002}

O Ambulatório de Oftalmologia (AMB) - HCFMUSP possui área útil de $624,24 \mathrm{~m}^{2}$, com 20 salas de consulta e funcionamento de 5 dias por semana, 12 horas por dia.

Para a estimativa dos custos fixos mensais do AMB HCFMUSP, foram inferidas despesas com:

a) recursos humanos: salários, encargos e benefícios da equipe não médica do AMB-HCFMUSP, composta por uma enfermeira, três auxiliares de enfermagem, quatro auxiliares de escritório e duas auxiliares de limpeza, além de 4 médicos oftalmologistas (2 médicos assistentes do HCFMUSP e 2 médicos residentes do HCFMUSP), com revezamento em duas duplas, por períodos de 6 horas por dia.

b) espaço físico e consumo de água e energia elétrica: O AMBHCFMUSP não possui gastos diretos com a utilização do espaço físico e com o consumo de água e energia elétrica. Estas despesas são custeadas e diluídas pelo custo operacional do "Complexo Hospitalar". Para a estimativa destes valores, foi considerado o custo proporcional de locação e de consumo de água e energia elétrica, de um imóvel de $128 \mathrm{~m}^{2}$, situado na mesma região que o HCFMUSP.

$O$ custo, por minuto, de uma sala do AMB-HCFMUSP, foi inferido, dividindo-se as despesas mensais estimadas, com espaço físico e com o consumo de água e energia elétrica, pelo número de salas existentes no local e pelo tempo de funcionamento por mês (20 dias $=240$ horas $=14.400$ minutos $)$.

$\mathrm{O}$ custo, por minuto, da equipe não médica do AMBHCFMUSP foi inferido, dividindo-se as despesas mensais estimadas com esta equipe pelo número de salas de consulta disponíveis no local e pelo período de trabalho em um mês (14.400 minutos).

$\mathrm{O}$ custo, por minuto, da equipe médica utilizada para o acompanhamento pós-operatório, em ambos os grupos (FACO e EECP), foi inferido, dividindo-se as despesas mensais estimadas com esta equipe pelo período de trabalho em um mês (14.400 minutos).

Estimou-se que, além do tempo necessário para a etapa da consulta, realizada exclusivamente pelo oftalmologista (biomicroscopia, tonometria e refratometria), o paciente tenha permanecido, em média, por mais 30 minutos no ambulatório, antes (admissão) e depois (marcação do retorno) de ser atendido pelo médico.

A estimativa da contribuição média de cada consulta pósoperatória, para o custo fixo da estrutura física e humana do AMB-HCFMUSP, foi inferida, considerando-se três variáveis:

a) despesa com a estrutura física.

b) despesa com os recursos humanos não médicos. 
c) despesa com os recursos humanos médicos.

O custo fixo médio, em ambos os grupos (FACO e EECP), do período pós-operatório completo, foi estimado, multiplicando-se o valor médio da consulta pelo número médio de retornos necessários, em ambos os grupos.

\section{Coleta de dados}

Fichas clínico-cirúrgicas foram preenchidas no momento da realização das consultas pré e pós-operatórias pelos médicos participantes do estudo, que contaram com a participação de um auxiliar de pesquisa.

\section{Processamento dos dados}

Os dados foram processados, utilizando-se o programa Access (Office 97).

\section{Procedimento para análise dos dados}

Todos os testes estatísticos foram realizados com auxílio do programa de computador SPSS (Statistical Package for Social Sciences) 10.0 para Windows ${ }^{\mathrm{TM}}$ (SPSS Inc, Chicago, IL, EUA).

\section{Análise estatística}

Valores de assimetria inferiores a um são considerados paramétricos e foram analisados estatisticamente pelo teste do QuiQuadrado para amostras independentes. Valores de assimetria maiores que um são considerados não-paramétricos e estudados estatisticamente pelos testes de Mann-Whitney.

Utilizou-se nível mínimo de significância estatística de 95\% ( $\mathrm{p}<0,05)$ em todos os testes aplicados.

\section{RESULTADOS}

\section{Seleção da amostra e seguimento dos sujeitos no decorrer do estudo}

O quadro 1 mostra o fluxo da seleção dos pacientes para inclusão no estudo e composição dos grupos.

\section{Eventos durante a cirurgia}

Os dados referentes aos tipos de anestesia utilizados para as cirurgias, em ambos os grupos, encontram-se na tabela 1 .

O tempo médio de permanência do paciente na sala cirúrgica, em ambos os grupos, é mostrado na tabela 2.

As cirurgias do grupo FACO foram realizadas por incisão corneal de, em média, 3,8 milímetros (mm) de extensão e sem sutura. As cirurgias do grupo EECP foram realizadas por incisão escleral de, em média, 11,1 $( \pm 1,0)$ mm de extensão, com 5,4 $( \pm 0,6)$ suturas, em média.

Foram realizadas, em média, 3,00 consultas pós-operatórias no grupo FACO e 5,17 no grupo EECP. Não foi realizada nenhuma consulta pós-operatória adicional no grupo FACO, enquanto que, no grupo EECP, nove sujeitos requisitaram uma consulta adicional, após remoção de sutura para controle do astigmatismo induzido pela cirurgia; um indivíduo compare-

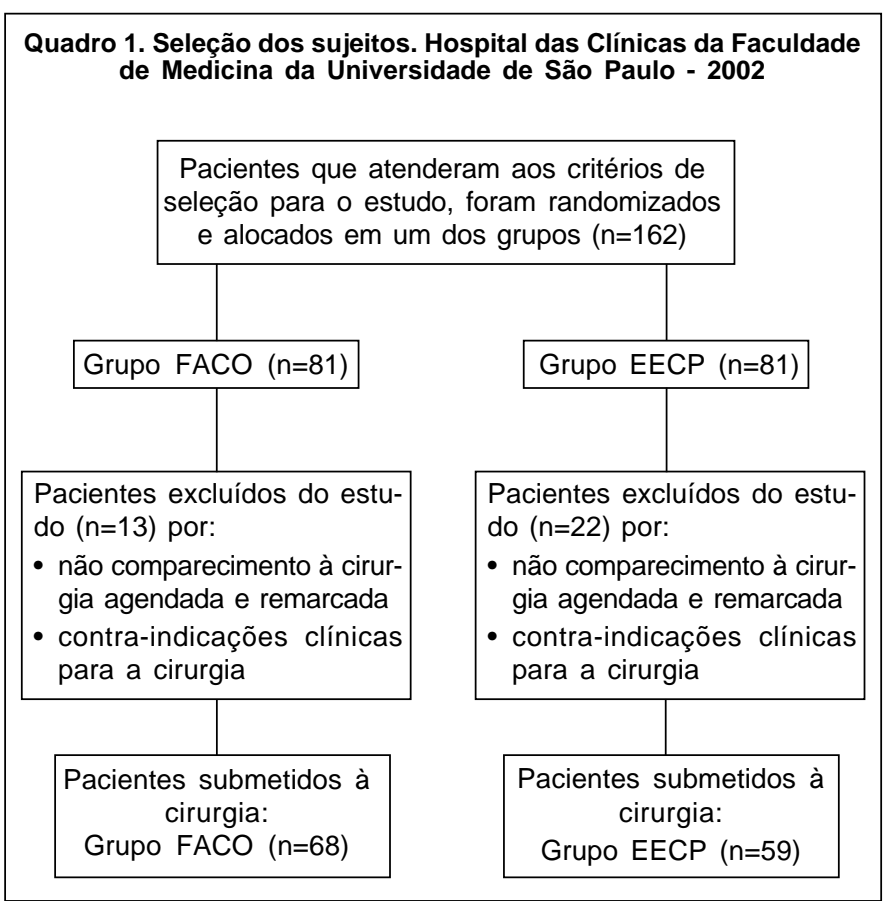

\begin{tabular}{|c|c|c|c|c|}
\hline \multirow[t]{2}{*}{ Anestesia } & \multicolumn{2}{|c|}{ FACO } & \multicolumn{2}{|c|}{ EECP } \\
\hline & $\mathbf{n}$ & $\%$ & $\mathbf{n}$ & $\%$ \\
\hline Tópica & 57 & 83,8 & 0 & 0,0 \\
\hline Bloqueio & 11 & 16,2 & 59 & 100,0 \\
\hline Total & 68 & 100,0 & 59 & 100,0 \\
\hline \multicolumn{5}{|c|}{$\chi^{2}=86,37 p<0,0001^{(*)}$} \\
\hline
\end{tabular}

\begin{tabular}{|c|c|c|c|}
\hline & $\begin{array}{c}\text { FACO } \\
n=68 \\
\text { média } \pm \text { d.p. }\end{array}$ & $\begin{array}{c}\text { EECP } \\
\mathrm{n}=59 \\
\text { média } \pm \text { d.p. }\end{array}$ & Teste \\
\hline $\begin{array}{l}\text { Tempo } \\
\text { (minutos) }\end{array}$ & $26 \pm 6$ & $35 \pm 10$ & $\begin{array}{r}U=678,5 \\
p=0,0001^{(*)}\end{array}$ \\
\hline
\end{tabular}

ceu em dia não programado por motivo não relacionado à cirurgia (trauma ocular, sem comprometimento visual).

O tempo médio de duração da etapa da consulta pósoperatória, realizada exclusivamente pelo oftalmologista, foi de aproximadamente 9 minutos, em ambos os grupos.

\section{Avaliação econômica}

Estima-se que o período útil de utilização do CCAHCFMUSP, para a realização das cirurgias, é de 10 horas por dia, 
o tempo médio de permanência do paciente na sala cirúrgica é de aproximadamente 26 minutos no grupo FACO e de aproximadamente 35 minutos no grupo EECP; o tempo médio necessário para a limpeza da sala após cada procedimento é de aproximadamente 10 minutos. Infere-se que, nas condições do estudo, possam ser realizadas 16 cirurgias do grupo FACO e 13 cirurgias do grupo EECP por dia, no CCA-HCFMUSP, 2002.

Os valores dos custos estimados das cirurgias e do acompanhamento pós-operatório, em ambos os grupos (FACO e EECP), são apresentados nas Tabelas 3 a 6.

$O$ custo fixo por cirurgia no grupo FACO foi estimado em $R \$$ 36,75 e no grupo EECP em $R \$ 39,95$. Esses valores foram obtidos dividindo-se os custos fixos totais do CCA-HCFMUSP (por sala/ dia), para ambos os grupos, pelo número estimado de cirurgias realizadas (por sala/dia), 16 no grupo FACO e 13 no grupo EECP.

\section{DISCUSSÃO}

Nos últimos anos, praticamente em todos os países desenvolvidos, tem ocorrido rápida mudança na técnica utilizada para a cirurgia de catarata, com aumento progressivo de cirurgias realizadas pela técnica de FACO. Porém, o emprego dessa técnica, no sistema público de saúde, acarreta aumento de custos, levando à diminuição da realização de outros procedimentos.

Para a implantação de um novo procedimento, é necessário estudo de sua eficiência, eficácia e efetividade. A eficiência de um procedimento é medida pela reação na qualidade de vida do paciente e pelo impacto econômico no sistema de saúde, pois novos gastos pressupõem redução de recursos destinados a outras áreas ${ }^{(44-45)}$.

Em aproximadamente $84 \%$ dos indivíduos do grupo FACO, a cirurgia foi realizada com anestesia tópica, em virtude da concordância entre a avaliação da equipe cirúrgica e a aceitação do paciente; nos demais casos, foi utilizado bloqueio peribulbar (Tabela 1). Em todos os sujeitos do grupo EECP, foi utilizado bloqueio peribulbar, devido à maior manipulação intra-ocular, principalmente da íris e ao mais prolongado tempo cirúrgico, fatores que, segundo alguns autores contraindicam a utilização da anestesia tópica ${ }^{(46-50)}$.

Realizou-se no grupo FACO incisão corneal, de 3,8 mm de extensão média, sem sutura e no grupo EECP, incisão escleral, de 11,1 mm de extensão média, com 5,4 suturas, em média.

Nessa pesquisa, o tempo médio de permanência do paciente na sala cirúrgica foi de $26( \pm 6)$ minutos no grupo FACO e de 35 ( \pm 10$)$ minutos no grupo EECP (Tabela 2). Estima-se que o tempo despendido antes do início da cirurgia para a acomodação do paciente e o preparo do material cirúrgico foi semelhante para ambos os grupos ou pouco maior no grupo FACO, assim como o tempo necessário para a remoção do paciente após a cirurgia. Desta maneira, infere-se que as cirurgias do grupo FACO foram, em média, aproximadamente $26 \%$ mais rápidas do que as do grupo $\mathrm{EECP}$, contribuindo para maior conforto do paciente e otimização do centro cirúrgico.

\begin{tabular}{|c|c|c|}
\hline Gastos & FACO (R\$) & $\operatorname{EECP}(R \$)$ \\
\hline $\begin{array}{l}\text { Insumos utilizados para o } \\
\text { preparo do paciente e } \\
\text { da equipe cirúrgica }\end{array}$ & 2,96 & 3,16 \\
\hline $\begin{array}{l}\text { Insumos utilizados para } \\
\text { limpeza da sala cirúrgica }\end{array}$ & 0,51 & 0,51 \\
\hline $\begin{array}{l}\text { Insumos utilizados para a } \\
\text { anestesia }\end{array}$ & 0,29 & 1,85 \\
\hline Insumos utilizados para a cirurgia & 464,65 & 254,44 \\
\hline Total & 468,92 & 259,96 \\
\hline
\end{tabular}

\begin{tabular}{|c|c|c|}
\hline $\begin{array}{l}\text { Gastos do CCA } \\
\text { (por sala/dia) }\end{array}$ & FACO (R\$) & EECP (R\$) \\
\hline Recursos humanos & 338,35 & 338,35 \\
\hline Depreciação de equipamentos & 130,00 & 61,25 \\
\hline $\begin{array}{l}\text { Espaço físico e consumo de } \\
\text { água e de energia elétrica }\end{array}$ & 119,78 & 119,78 \\
\hline Total & 588,13 & 519,38 \\
\hline
\end{tabular}

A maior eficiência no uso da estrutura física e dos recursos humanos já existentes (capacidade instalada), com aumento do número de cirurgias, reduz o custo individual da cirurgia $^{(26,50-51)}$.

No presente estudo, a avaliação econômica foi realizada, considerando-se a eficiência total da unidade cirúrgica, condizente com a realidade do HCFMUSP para este momento, em que condições gerenciais e ambientais estimulam a dedicação de médicos e pessoal auxiliar envolvidos, propiciando bom rendi- 


\begin{tabular}{|c|c|c|}
\hline \multicolumn{3}{|c|}{$\begin{array}{l}\text { Tabela 6. Estimativa dos custos de cada procedimento, em ambos } \\
\text { os grupos (FACO e EECP). Hospital das Clínicas da Faculdade de } \\
\text { Medicina da Universidade de São Paulo - } 2002\end{array}$} \\
\hline Custos & FACO (R\$) & EECP (R\$) \\
\hline \multicolumn{3}{|l|}{ Cirúrgicos } \\
\hline Custos variáveis & 468,92 & 259,96 \\
\hline Custos fixos & 36,75 & 39,95 \\
\hline Período pós-operatório & 22,68 & 39,08 \\
\hline Total & 528,35 & 338,99 \\
\hline
\end{tabular}

mento do serviço de Oftalmologia. Assim, foram realizadas no HCFMUSP, de janeiro a novembro de 2002, em média, 378 cirurgias de catarata por mês (Universidade de São Paulo, 2002).

Nessa investigação, como parte dos dados utilizados para o cálculo dos custos são estimativas, considera-se que este modelo tenha sido construído como uma simulação, assemelhando-se o máximo possível à realidade do HCFMUSP, em 2002.

Os gastos estimados com os insumos utilizados para as cirurgias (custos variáveis), em ambos os grupos (FACO e EECP), foram de R $\$ 468,92$ no grupo FACO e R $\$ 259,96$ no grupo EECP, a diferença de $\mathrm{R} \$ 208,96$ reflete despesas com materiais mais dispendiosos, utilizados para as cirurgias no grupo FACO. Os gastos com os insumos para a cirurgia aumentaram cerca de $80 \%$ quando a técnica de FACO foi utilizada (Tabela 3). A possível diminuição das despesas com insumos e materiais descartáveis, quando comparadas com outros hospitais e clínicas, deve-se a medidas, como aquisição de materiais em concorrência pública em grandes quantidades e o uso máximo de insumos (colírios e substâncias anestésicas são utilizados em mais de um procedimento).

Os gastos com a manutenção do CCA-HCFMUSP (custos fixos) são semelhantes para ambos os grupos; porém, como as cirurgias do grupo FACO requerem aparelhagem específica (facoemulsificador), o custo operacional da sala cirúrgica destinada à realização das cirurgias deste grupo foi acrescido do custo de depreciação do equipamento. Estimando-se que o período útil do CCA-HCFMUSP para a realização das cirurgias é de 10 horas por dia, o tempo médio de permanência do paciente na sala cirúrgica é de aproximadamente 26 minutos no grupo FACO e de aproximadamente 35 minutos no grupo EECP e o tempo necessário para a limpeza da sala após cada procedimento é de aproximadamente 10 minutos, infere-se que, nas condições do estudo, possam ser realizadas 16 cirurgias do grupo FACO e 13 cirurgias do grupo EECP por dia, no CCA-HCFMUSP. Estima-se que, no grupo FACO, a eficiência do CCA-HCFMUSP foi $23 \%$ maior que a do grupo EECP. A otimização da unidade cirúrgica, no grupo FACO diminui o custo fixo de cada procedimento, uma vez que a despesa operacional do CCA é a mesma, independente do número de procedimentos realizados. Infere-se que, a partir dos dados apresentados na tabela 4 , a economia com a otimização do CCA, no grupo FACO, não só compensou o capital investido com o equipamento adicional como também tornou o custo fixo de cada procedimento por FACO inferior ao por EECP.
No tocante ao acompanhamento pós-operatório, estimou-se que a despesa do HCFMUSP com cada consulta de retorno foi de aproximadamente $\mathrm{R} \$ 7,66$ (Tabela 5). Considerando-se que o grupo FACO necessitou, em média, de 3 retornos pós-operatórios e o grupo EECP, em média, de 5,17 retornos, infere-se que as despesas hospitalares com o acompanhamento pós-operatório sejam, em média, R \$ 16,40 (42\%), menores no grupo FACO.

Estima-se que as despesas associados à cirurgia e ao período pós-operatório no grupo FACO, após 3 meses da cirurgia, no HC-FMUSP em 2002, foram, em média, de $\mathrm{R} \$ 528,35$, enquanto que no grupo EECP, foram de $\mathrm{R} \$ 338,99$. Os gastos totais no grupo FACO foram, em média, $\mathrm{R} \$ 189,07$ (56\%), maiores do que no grupo EECP (Tabela 6).

O reembolso do SUS para a cirurgia da catarata por FACO com implante de LIO dobrável é de R \$643,00 e o por EECP com LIO rígida é de R \$422,00 (Secretaria da Saúde, 2002). A diferença de reembolso entre ambos os procedimentos é de $\mathrm{R} \$ 221,00$. Assim sendo, estima-se que, para as condições do HCFMUSP, em 2002, a realização da cirurgia por FACO, em larga escala, é economicamente vantajosa, uma vez que a diferença entre a receita e a despesa com cada procedimento é, em média, de $\mathrm{R} \$ 114,65$ na FACO e de R \$ 83,01 na EECP. No momento atual, em que o SUS passa a financiar a realização da cirurgia de catarata por FACO, torna-se imperativo avaliar a exeqüibilidade econômica desse procedimento. O presente estudo ressalta a exeqüibilidade da cirurgia por FACO no HCFMUSP, em 2002.

Considera-se, também, que o reembolso do SUS para cada consulta pós-operatória com tonometria (apenas do olho operado) é de R \$ 5,92 e que os gastos do HCFMUSP com cada retorno são estimadas em $\mathrm{R} \$ 7,66$. Nessas condições, em que a despesa com os retornos pós-operatórios é superior à receita, é, também, evidenciada a vantagem econômica para o HCFMUSP, com a utilização da FACO, por requerer menor número de consultas após a cirurgia.

Embora alguns autores relatem que a utilização de tecnologia sofisticada, aplicada na promoção da saúde ocular no Sistema Público de países em desenvolvimento, seja inviável, especialmente com relação à cirurgia da catarata, a qual consome uma expressiva parcela do orçamento destinado à saúde pública, o presente estudo demonstrou que, para a realidade do HCFMUSP, em 2002, a técnica de FACO é economicamente viável, oferecendo vantagens econômicas para o paciente e para o hospital ${ }^{(19,32,49)}$.

Com maior tempo de seguimento desses pacientes, talvez seja possível confirmar para a realidade do SUS vantagens econômicas favoráveis ao emprego da FACO em relação à EECP, à semelhança do que descreveram para a Inglaterra ${ }^{(32)}$.

Os aspectos econômicos são importantes instrumentos para nortear as decisões políticas. Porém, a saúde do idoso, também, deve ser considerada um bem econômico e ser valorizada pela sociedade.

Economia em saúde não se faz gastando menos, mas gastando-se melhor, isto é, obtendo-se os maiores benefícios sociais aos menores custos incorridos em sua consecução ${ }^{(46)}$. A utilização da moderna tecnologia para a cirurgia da catarata 
em países em desenvolvimento deve resolver mais problemas do que criá-los ${ }^{(52)}$.

Além disso, considera-se que uma importante contribuição do presente estudo seja a elaboração de um modelo de avaliação econômica, baseado na realidade do HCFMUSP, em 2002. O emprego desse modelo, para a avaliação dos gastos associados à cirurgia de catarata por outros centros oftalmológicos de diferentes regiões do país, pode fornecer subsídios para a implantação e o acompanhamento de importantes ações de saúde ocular.

\section{CONCLUSÕES}

Nas condições propostas para este estudo, conclui-se que os custos totais da cirurgia de catarata mediante a técnica de FACO são maiores do que os com a técnica de EECP; e a remuneração efetuada pelo Sistema Único de Saúde ao HCFMUSP confere à técnica por FACO maiores vantagens econômicas do que a técnica por EECP.

\section{ABSTRACT}

Purpose: To evaluate the clinical and economic aspects of cataract surgery by phacoemulsification, when compared to extracapsular extraction. Methods: A prospective study on 162 subjects was carried out at the "Hospital das Clínicas", Medical School of the University of São Paulo in 2002. The subjects who had cataract were randomized for surgery by phacoemulsification and by extracapsular extraction. Results: In this study, phacoemulsification technique allowed the use of local anesthesia. The number of follow-up visits and the postoperative time offered quick visual restoration and clinical discharge. The use of phacoemulsification in the "Hospital das Clínicas" of the Medical School of the University of São Paulo contributed to quick surgery, optimized the surgery theater and showed cost-effective advantages for the hospital. Conclusion: According to the conditions of the "Hospital das Clínicas" of the Medical School of the University of São Paulo, we concluded that the total costs of cataract surgery by phacoemulsification are higher than those by extracapsular extraction, and surgery fees offered by the Public Health Service to the "Hospital das Clínicas" of the Medical School of the University of São Paulo have shown more cost-effective advantages for phacoemulsification than for extracapsular extraction.

Keywords: Cataract extraction/methods; Cataract extraction/ economics; Phacoemulsification/methods; Phacoemulsification/economics; Blindness/prevention \& control; Economic factors; Hospitals, teaching; Comparative study

\section{REFERÊNCIAS}

1. Javitt JC, Wang F, West SK. Blindness due to cataract: epidemiology and prevention. Annu Rev Public Health 1996;17:159-77.
2. Kupfer C. The international agency for the prevention of blindness. Am $\mathbf{J}$ Ophthalmol 1994;117:253-7.

3. Ellwein LB, Kupfer C. Strategic issues in preventing cataract blindness in developing countries. Bull World Health Organ 1995;73:681-90.

4. Thylefors B, Négrel AD, Pararajasegaram R, Dadzie KY. Global data on blindness. Bull World Health Organ 1995;73:115-21.

5. Alves MR, Kara-José N. Catarata: um problema de saúde pública. In: KaraJosé N, Delgado AMN, Arieta CEL, Rodrigues MLV, Alves MR, editores. Prevenção da cegueira por catarata. Campinas: UNICAMP; 1996. p.11-8.

6. Kara-José N, Barbosa E, Fonseca-Neto JC, Oura MH, Martins WH. Considerações sobre aspectos sociais do atendimento clínico e cirúrgico de pacientes portadores de catarata senil. Arq Bras Oftalmol 1982;45:115-8.

7. Sommer A. Organizing to prevent third word blindness. Am J Ophthalmol 1989;107:544-6.

8. Arieta CEL, Kara-José N, Alves MR. Estratégias para a ação contra a catarata. In: Kara-José N, Delgado AMN, Arieta CEL, Rodrigues MLV, Alves MR, editores. Prevenção da cegueira por catarata. Campinas: UNICAMP; 1996. p.19-35.

9. Kara-Jose N, Delgado NA, Arieta CEL. Catarata. In: Rodrigues MLV, editor. Oftalmologia clínica. Rio de Janeiro: Cultura Médica; 1992. p.288-302.

10. Temporini ER, Kara-José N, Kara-Junior N. Catarata senil: características e percepções de pacientes atendidos em projeto comunitário de reabilitação visual. Arq Bras Oftalmol 1997;60:79-83.

11. Kara-José N, Temporini ER. Cirurgia de catarata: o porquê dos excluídos. Rev Panam Salud Publica 1999;6:242-8.

12. Kara-Junior N, Temporini ER, Kara-Jose N. Cataract surgery: expectations of patients assisted during a community project in São Paulo, State of São Paulo, Brazil. Rev Hosp Clín Fac Med S Paulo 2001;56:163-8.

13. Temporini ER, Kara-Junior N, Holzchuh N, Kara-José N. Popular beliefs regarding the treatment of senile cataract. Rev Saúde Pública 2002;36:343-9.

14. Leite F. Convênio gera até $51 \%$ da renda de hospital. Folha de São Paulo, 2002; 22 jul. C-1.

15. Kara-Junior N, Arieta CEL. Catarata senil. In: Kara-Jose N, Almeida GV, editores. Senilidade ocular. São Paulo: Roca; 2001. p.99-107.

16. Foster A. Como podemos eliminar a cegueira por cataratas? In: Curso Internacional de saúde ocular comunitária, Teresina, 2002. Cristian Blind Mission (CBM) Internacional, 2002. p.12-22.

17. Kara-José N, Contreras F, Campos MA, Delgado AMN, Mowery RL, Ellwein LB. Screening and surgical intervention results from Cataract-freezone projects in Campinas, Brasil and Chimbote, Peru. Int Ophthalmol 1990; 14:155-64.

18. Kara-Junior N, Schellini SA, Silva MRBM, Bruni LF, Almeida AGC. Projeto Catarata - Qual a sua importância para a comunidade? Arq Bras Oftalmol 1996;59:490-6.

19. Schwab L. Eye care delivery in developing nations: paradigms, paradoxes, and progress. Ophthalmic Epidemiol 1994;1:149-54.

20. Kara-Jose N, Temporini ER. Catarata e cegueira: epidemiologia e prevenção. In: Arieta CEL, editor. Cristalino e catarata. Rio de Janeiro: Cultura Médica; 2002. p.49-56.

21. Berton JF, Pasquinelli FF, Pinto-Junior W, Fonseca JC, Kara-José N. As vantagens da alta precoce após cirurgia da catarata. Arq Bras Oftalmol $1980 ; 43: 17-8$.

22. Kara-Jose N, Delgado AMN, Arieta CEL. Exeqüibilidade da cirurgia de catarata em hospital-escola: em busca de um modelo econômico. Rev Assoc Med Bras 1994;40:186-8.

23. Arieta CEL, Taiar A, Kara-José N. Utilização e causas de suspensão de intervenções cirúrgicas oculares em centro cirúrgico ambulatorial universitário. Rev Assoc Med Bras 1995;41:233-5.

24. Arieta CEL, Kara-José N. Otimização de recursos e cirurgia ambulatorial de catarata. In: Kara-José N, Delgado AMN, Arieta CEL, Rodrigues MLV, Alves MR, editores. Prevenção da cegueira por catarata. Campinas: UNICAMP; 1996. p.37-44.

25. Delgado AMN, Kara-Jose N. Projetos comunitários em oftalmologia. In: KaraJosé N, Delgado AMN, Arieta CEL, Rodrigues MLV, Alves MR, editores. Prevenção da cegueira por catarata. Campinas: UNICAMP;1996. p.55-69.

26. Arieta CEL, Kara-José N, Carvalho-Filho DM, Alves MR. Optimization of a university cataract-patient care service in Campinas, Brasil. Ophthalmic Epidemiol 1999;6:113-23.

27. Yorston Y. Are intraocular lenses the solution to cataract blindness in Africa? Br J Ophthalmol 1998;82:469-71.

28. West S, Sommer A. Prevention of blindness and priorities for the future [comment on Bull World Health Organ 2002;80:419]. Bull World Health Organ 2001;79:244-9. 
29. Taylor HR, Sommer A. Cataract surgery. A global perspective. Arch Ophthalmol 1990;108:797-8.

30. Ohrloff C, Zubcov AA. Comparison of phacoemulsification and planned extracapsular extraction. Ophthalmologica 1997;211:8-12.

31. Albanis C, Dwyer MA, Ernest T. Outcomes of extra-capsular cataract extraction and phacoemulsification performed in a University training program. Ophthalmic Surg Lasers 1998;29:643-8.

32. Minassian DC, Rosen P, Dart JK, Reidy A, Desai P, Sidhu M, et al. Extracapsular cataract extraction compared with small incision surgery by phacoemulsification: a randomized trial [Comment on Br J Ophthalmol 2001;85:765-6].. Br J Ophthalmol 2001;85:822-9.

33. Leinoken J, Laatikainen L. Changes in visual acuity of patients undergoing cataract surgery during the last two decades. Acta Ophthalmol Scand 2002;80:506-11.

34. Leaming DV. Practice styles and preferences of ASCRS members-1998 Survey. J Cataract Refract Surg 1999;25:851-9.

35. The Royal College of Ophthalmologists. Cataract surgery guidelines. London: Royal College of Ophthalmologists; feb. 2001. 21p. [ cited 2003 Nov 20].. Available from: http://www.rcophth.ac.uk/publications/guidelines/ cataract_surgery2.html

36. Lundstrom M, Stenevi U, Thorburn W. The Swedish National Cataract Register: a 9-year review. Acta Ophthalmol Scand 2002;80:248-57.

37. Andrade C, Farah EE, Mendonça FLP, Taiyuwa N, Paes-Junior U. Cálculo de custos para consultórios. São Paulo: Quest, 1999.

38. Vargas GP. Cirurgia ambulatorial em clínicas oftalmológicas: empreendimento viável? Oftalmol Foco 2002;80:42-3.

39. Castells X, Comas M, Castilla M, Cots F, Alarcón S. Clinical outcomes and cost of cataract surgery performed by planned ECCE and phacoemulsification. Int Ophthalmol 1998;22:363-7.
40. Heslin KB, Guerriero PN. Clinical retrospective study comparing planned extracapsular cataract extraction and phacoemulsification with and without lens implantation. Ann Ophthalmol 1984;16:956-62.

41. Sheets JH. A step beyond ECCE. CLAO J 1987;13:67-70

42. Schein OD, Steinberg EP, Javitt JC, Cassard SD, Tielsch JM, Steinwachs DM, et al. Variation in cataract surgery practice and clinical outcomes [commented on Ophthalmology 1994;101:977-8]. Ophthalmology 1994;101:1142-52.

43. Ionides A, Claoué C. Resource management of cataract patients: can visual rehabilitation be achieved in three visits? J Cataract Refract Surg 1996;22:717-20.

44. Medice AC. Economia e financiamento do setor de saúde no Brasil [tese]. São Paulo, Faculdade de Saúde Pública da Universidade de São Paulo, 1994.

45. American Academy Of Ophthalmology. International ophthalmology. San Francisco, 2002. 369p.

46. Dunlop JM. Public health in third millennium. Public Health 1995;109:165-7.

47. Roper-Hall MJ. Cataract: a world problem. Ophthalmic Surg 1988;19:393-4.

48. Chang DF. Factoring cost, is phacoemulsification still the procedure of choice? Br. J. Ophthalmol 2001:85:765-6.

49. Patel CKB, Burns TA, Crandall A, Shomaker ST, Pace NL, Eerd A, Clinch T. A comparison of topical and retrobulbar anesthesia for cataract surgery. Ophthalmology 1996;103:1196-203.

50. Kara-Jose N, Arieta CEL. South America Programme: Brazil. Community Eye Health 2000;13(36):55-6.

51. Taylor HR, Sommer A. Cataract surgery. A global perspective. Arch Ophthalmol 1990;108:797-8.

52. Gillies M, Brian G, Nauze JL. Modern surgery for global cataract blindness. Arch Ophthalmol 1998;116:90-2.

\title{
XXV CONGRESSO DO HOSPITAL SÃO GERALDO
}

\section{1 a 13 de novembro de 2004 Belo Horizonte - MG}

\author{
INFORMAÇÕES: Consult Comunicação e Eventos \\ Fone/ Fax: (31) 3274-1550 \\ e-mail: comunica@consultcom.com.br
}

